\section{Cricoid pressure}

To the Editor:

I feel that Jean-Francois Hardy, in his recent review "Large volume gastro-esophageal reflux: a rationale for risk reduction in the perioperative period," described some of the literature on cricoid pressure but did not subject it to critical analysis. I am concerned that advising readers that "cricoid pressure is simple" encourages the wrong attitude and approach. Moreover can its 100 per cent effectiveness be taken for granted? Complacency about such subjects surely needs to be avoided.

For a time I followed Selwyn Crawford's technique of applying cricoid pressure prior to the induction of anaesthesia but I gave up this approach when a patient hiccoughed, as the thiopentone circulated, and thereby filled up her pharynx with fluid from the stomach with resulting aspiration and subsequent death a few hours later. More recently, in a case where cricoid pressure was applied, an easy and simple intubation was converted into an impossible one; laryngoscopy displayed only the epiglottis with its base resting on the posterior wall of the pharynx. Last week I was told of a colleague who was sure that cricoid pressure was properly applied but upon inserting the laryngoscope found the pharynx full of fluid.

Such examples bring home the pitfalls facing anaesthetists with each and every laryngoscopy, intubation and application of cricoid pressure and the degree of concentration and dedication required if perfection is to be achieved in their performance every time. Moreover, although such incidents occur few of them seem to get as far as being published which can give us all a false impression.

Thank you for your interest.

R. Williamson BSc MBChB DA FFARCS

Senior Specialist/Senior Lecturer (Anaesthetics)

University of Natal

South Africa

\section{$R E P L Y$}

Sellick's manoeuvre is a simple concept, sometimes difficult to implement in everyday practice. Despite the sophisticated equip. ment used in contemporary anaesthesia, proper application of cricoid pressure remains based on sound clinical judgment and experience. It is, in effect, a form of medical art. Though not always easy to achieve, a smooth induction of anaesthesia and perfect timing of cricoid compression are of the utmost importance to avoid either provoking active vomiting or passive regurgitation.

I believe Sellick's recommendations still hold rue. Judiciously applied, these will effectively preveni gastrooesophageal reflux in a majority of patients at risk of regurgitating on induction of anaesthesia. However close to perfection, I do not believe any one single measure will ever prevent gastrooesophageal reflux in 100 per cent of cases. But, is this not inherent to the practice of medicine?

Jean-Francis Hardy MD

Montreal, Que.

\section{Interpleural or paravertebral block at thoracotomy?}

\section{To the Editor:}

I am writing in response to the Editorial "Interpleural block" published in the Journal in March 1989.

Dr. Lewis refers to the "open chest" technique used by Kambam JR et al. ${ }^{1}$ whereby an epidural catheter is inserted into the chest at thoracotomy to provide access to the pleural cavity for postoperative infusion of bupivacaine. He suggests that this technique offers an alternative to more established postthoracotomy analgesic methods such as epidural, parenteral narcotics or intercostal nerve blocks. He does not mention paravertebral block, which we have recently described as an alternative "open chest" technique ${ }^{2}$ placing the epidural catheter in the paravertebral space under direct vision via an extrapleural route. We believe that paravertebral block attained thereby is the method of choice for a number of reasons.

The anaesthetic is delivered to the most logical space through which runs the intercostal nerve as it exits the intervertebral foramen, its dorsal primary ramus (supplying posterior spinal muscles and costovertebral ligaments strained at thoracotomy), its collateral branch (supplying the parietal pleura), and the sympathetic afferents on that side (supplying the visceral pleura). Inhibition of the endocrine-metabolic stress response to operative trauma requires effective sympathetic and somatic block. ${ }^{3}$

We have demonstrated radiologically that the infusion using this technique is confined to the paravertebral space by the fifth day of infusion. ${ }^{4}$ Paravertebral infusion of local anaesthetic agents does not suffer the disadvantages of interpleural infusion after thoracotomy, namely loss via the chest tube, dilution by irrigation fluid or blood, binding to blood proteins in a haemothorax, or rapid absorption by damaged lung quoted as possible explanations for unsatisfactory analgesia. ${ }^{1}$ Paravertebral infusion is not contraindicated in pneumonectomy patients who are unsuitable for interpleural block. It is unlikely to affect the phrenic nerve, and should only produce a unilateral sympathetic block. Paravertebral block should provide adequate analgesia for anterior thoracotomies where interpleural block may not be effective.'

In a randomised controlled trial, the "open chest" 